\title{
Importância da oftalmoscopia realizada na Atenção Básica de Saúde para diagnóstico precoce da Retinopatia Diabética e Hipertensiva
}

\section{Importance of ophthalmoscopy performed in Primary Health Care for early diagnosis of Diabetic and Hypertensive Retinopathy}

Tiana Carneiro Simões de Almeida ${ }^{* 1}$, Bárbara Marcias de Sousa², Gisele Roque de Souza ${ }^{3}$, Thomás Alves Gomes ${ }^{4}$, Lívia Oliveira Delgado Mota ${ }^{5}$

Como citar esse artigo. Almeida

T. C. S; Sousa, B. M.; Souza, G R; Gomes, T. A; Mota, L. O. D Importância da oftalmoscopia realizada na Atenção Básica de Saúde para diagnóstico precoce da Retinopatia Diabética e Hipertensiva. Revista de Saúde 2021 Ago/Nov.; 12 (3): 33-36.

\section{Resumo}

A Hipertensão Arterial Sistêmica (HAS) e o Diabetes Mellitus (DM) fazem parte do rol de Doenças Crônicas Não Transmissíveis (DCNT) mais comuns e com a maior taxa de morbimortalidade, atualmente, no Brasil e no mundo. Diversas complicações estão atreladas a essas patologias dentre elas pode-se destacar a Retinopatia Diabética (RD) e Hipertensiva (RH), sendo a primeira uma causa importante de cegueira adquirida e irreversível no mundo. O presente estudo tem o objetivo de mostrar de forma analítica a importância da realização da Oftalmoscopia pelo médico da Atenção Básica à saúde, à fim de promover um diagnóstico precoce da retinopatia diabética e hipertensiva. Trata-se de uma revisão de literatura, na qual foram incluídos 21 artigos do Scielo, PubMed, Cochrane Libary, Diretrizes brasileiras de HAS e DM, além de dados da OMS e Cadernos e Guias do Ministério da Saúde. A RD e RH estão interligadas ao controle deficitário da glicemia e da pressão arterial. O médico generalista representa uma imagem de suma importância quando se emprega precocemente o exame de fundo de olho nas suas consultas de rotina ambulatorial dentro da Atenção Básica. O Médico da atenção primária da saúde deve estar preparado para aplicação de condutas precoces e exatas, como a realização da oftalmoscopia direta, para viabilizar a redução de danos como a cegueira e gastos públicos. Por fim, é evidente a real necessidade da implementação do aprendizado e realização do exame de fundoscopia pelos profissionais médicos da Unidade Básica de Saúde.

Palavras-chave: Oftalmoscopia; Hipertensão arterial; Retinopatia diabética; Atenção Básica

\begin{abstract}
Systemic Arterial Hypertension (SAH) and Diabetes Mellitus (DM) are part of the most common list of Chronic Noncommunicable Diseases (NCDs) and the highest rate of morbidity and mortality, currently, in Brazil and worldwide. Several complications are linked to these pathologies, including Diabetic Retinopathy (RD) and Hypertension (RH), the first being an important cause of acquired and irreversible blindness in the world. This study aims to show analytically the importance of performing Ophthalmoscopy by the Primary Health Care physician, in order to promote an early diagnosis of diabetic and hypertensive retinopathy. This is a literature review, which included 21 articles from Scielo, PubMed, Cochrane Libary, Brazilian guidelines for SAH and DM, in addition to WHO data and Ministry of Health Notebooks and Guides. RD and RH are interconnected deficient control of blood glucose and blood pressure. The general practitioner represents an image of paramount importance when the eye fundus exam is used early in his routine outpatient consultations within Primary Care. The primary health care physician must be prepared to apply early and accurate procedures, such as performing direct ophthalmoscopy, to make it possible to reduce damage such as blindness and public spending. Finally, the real need to implement learning and carry out the fundus examination by the medical professionals of the Basic Health Unit is evident.
\end{abstract}

Keywords: Ophtalmoscopy; Arterial hypertension; Diabetic retinopathy; Primary Care

\section{Introdução}

A Hipertensão Arterial Sistêmica (HAS) e o Diabetes Mellitus (DM) fazem parte do rol de Doenças Crônicas Não Transmissíveis (DCNT) mais comuns e com a maior taxa de morbimortalidade, atualmente, no Brasil e no mundo. Elas representam um grande entrave à atuação do Sistema Único de Saúde (SUS) por requererem um acompanhamento contínuo e prolongado, aliada a elevada complexidade de suas complicações, o que onera os cofres públicos e gera desafios a atuação dos diversos setores da saúde, principalmente da Atenção Básica. ${ }^{1}$

De acordo com dados da Organização Mundial da Saúde (OMS) registrados no VIGITEL Brasil de 2019 (Vigilância de Fatores de Risco e Proteção para Doenças Crônicas por Inquérito Telefônico), no ano de 2016, 71\% das 57 milhões de mortes totais no mundo foram por DCNT, e no Brasil, nesse mesmo ano, essa representação foi de $74 \%$ dos óbitos totais, sendo que

\footnotetext{
Afiliação dos autores:

1*Discente do Curso de Medicina, Universidade de Vassouras, Vassouras, Rio de Janeiro, Brasil, ORCID: https://orcid.org/0000-0001-7001-8933

${ }^{2}$ Discente do Curso de Medicina, Universidade de Vassouras, Vassouras, Rio de Janeiro, Brasil, ORCID: https://orcid.org/0000-0001-6979-2630

${ }^{3}$ Discente do Curso de Medicina, Universidade de Vassouras, Vassouras, Rio de Janeiro, Brasil, ORCID: https://orcid.org/0000-0003-4450-8052

${ }^{4}$ Discente do Curso de Medicina, Universidade de Vassouras, Vassouras, Rio de Janeiro, Brasil, ORCID: https://orcid.org/0000-0003-0122-2273

${ }^{5}$ Docente do Curso de Medicina, Universidade de Vassouras, Vassouras, Rio de Janeiro, Brasil, ORCID: https://orcid.org/0000-0001-7147-0171 
$28 \%$ são das doenças cardiovasculares e $5 \%$ por DM. $^{2}$ Esse conjunto de doenças acomete todas as classes socioeconômicas, porém a sua mortalidade é ainda maior nos países onde há uma média e baixa renda per capita, e tem como os mais predispostos os idosos e os pacientes de baixa escolaridade. ${ }^{3}$

$\mathrm{O}$ diabetes mellitus (DM) é caracterizado por um quadro de hiperglicemia, a qual pode ocorrer como resultado de uma destruição autoimune das células betapancreáticas, produtoras do hormônio hipoglicemiante insulina (Diabetes mellitus tipo 1- DM 1), ou pela resistência insulínica, a causa mais comum (Diabetes mellitus tipo $2-\mathrm{DM}$ 2)4. De acordo com a Diretriz da Sociedade Brasileira de Diabetes 2019-2020, no cenário atual, percebe-se ainda um crescente aumento da prevalência de diabetes mellitus, o que está associado a fatores de risco como: a mudança do hábito alimentar, sedentarismo, a obesidade, o processo de urbanização, o envelhecimento da sociedade e ao aumento da sobrevida dos pacientes diabéticos. ${ }^{5}$

A Hipertensão Arterial Sistêmica (HAS) é definida pela $7^{\text {a }}$ Diretriz de Hipertensão Arterial da Sociedade Brasileira de Cardiologia como sendo uma elevação sustentada da pressão arterial sistólica (PAS) $\geq 140 \mathrm{mmHg}$ e/ou pressão arterial diastólica (PAD) $\geq 90 \mathrm{mmHg}$. Ela é uma condição clínica multifatorial, correlacionadaadiversosdistúrbioscomoosmetabólicos, levando a lesões de órgãos-alvos, a exemplo dos rins, olhos, do cérebro e do próprio coração, provocando alterações tanto funcionais quanto estruturais. A sua prevalência no Brasil é maior na população masculina e idosa, principalmente na faixa etária $\geq 75$ anos de idade. $^{6}$

As doenças crônicas, como no caso da HAS e do DM, são fatores de risco para o desenvolvimento de doenças do aparelho circulatório. Juntas são responsáveis por uma elevada morbimortalidade e um alto nível de incapacitação da população brasileira, principalmente naquela em idade produtiva. Diversas complicações estão atreladas a essas patologias, dentre elas têm-se as vasculopatias, e as neurológicas, nas quais pode-se destacar a Retinopatia Diabética e Hipertensiva, sendo a primeira uma importante causa de cegueira adquirida e irreversível no mundo. ${ }^{1}$

A Retinopatia Diabética (RD) é uma complicação microvascular relacionada ao inadequado controle da hiperglicemia e ao curso crônico dessa doença. Esperase que em 20 anos, $99 \%$ dos pacientes com DM1 e $60 \%$ daqueles com DM2 apresentem alguma evolução para retinopatia ${ }^{1}$. Perante a essa problemática, é de extrema relevância um acompanhamento oftalmológico e a realização de uma avaliação do fundo de olho a partir de um exame chamado de Oftalmoscopia, o qual quando realizado de forma precoce reduz muito a incidência dessa complicação. Por isso, pacientes portadores de DM 1 devem realizar seu primeiro exame oftalmológico
5 anos após o diagnóstico, enquanto que pacientes com DM 2 devem realizar esse acompanhamento mediante o seu diagnóstico. ${ }^{7}$ Em relação a Retinopatia Hipertensiva $(\mathrm{RH})$ a vascularização da retina sofrerá alterações da sua conformação, com formação de tortuosidades, uma lesão precoce que está relacionada a constrição generalizada das arteríolas, com redução do calibre arteriovenoso fisiológico, consequente também a um inadequado controle terapêutico e da pressão arterial. ${ }^{8}$

Frente ao cenário enfrentado, é notória a necessidade de ações de rastreamento precoce e definitivo para o diagnóstico da Retinopatia Diabética e Hipertensiva, a partir do manejo da Oftalmoscopia pelos médicos da Atenção Básica, trazendo à tona a atuação mais efetiva do Sistema Único de Saúde (SUS) e de todos os seus setores no preparo desses profissionais, integrando o cuidado, promovendo e protegendo a saúde da população suscetível. ${ }^{9}$

O objetivo deste referido estudo foi mostrar de forma analítica dados que elucidam a importância da realização da Oftalmoscopia pelo médico da Atenção Básica à saúde, à fim de promover um diagnóstico precoce da retinopatia diabética e hipertensiva, reduzindo as chances de cegueira irreversível e de incapacitação desses pacientes, impulsionando uma maior autonomia no seu processo de autocuidado, permitindo assim uma melhor qualidade de vida, além de minimizar a oneração ao SUS proveniente de suas complicações.

\section{Metodologia}

Trata-se de uma pesquisa bibliográfica de abordagem qualitativa e caráter descritivo. Cabe ressaltar que a pesquisa bibliográfica foi desenvolvida com auxílio de material já elaborado, constituído principalmente de livros e artigos científicos ${ }^{1}$.

Os dados foram coletados em Base de Dados Virtuais. Para tal utilizou-se a Biblioteca Virtual de Saúde (BVS), na seguinte Base de informação: Scientific Eletrônic Library Online (Scielo), National Library of Medicine (PubMed) e Cochrane Libary, além das Diretrizes da Sociedade Brasileira de Diabetes e Hipertensão, dados do site oficial da Organização Mundial da Saúde e Cadernos e Guias do Ministério da Saúde, no período de 2008 à 2019. Foram selecionados um total de 52 artigos, sendo que 31 deles entraram no processo de exclusão por não abrangerem a importância da realização da oftalmoscopia direta pelo médico generalista em uma Unidade Básica de Saúde, pelo fato de alguns apresentarem ideias repetidas dos outros artigos já selecionados e por serem trabalhos muito antigos. No entanto, 21 artigos foram incluídos para elaboração desse trabalho, pois relataram de forma precisa os temas abordados nesse trabalho, trazendo um arsenal de informações como dados epidemiológicos que 
foram necessários para a montagem desse manuscrito

A estratégia de pesquisa utilizada para busca dos artigos foi: "Retinopatia Diabética" AND "Hipertensão Arterial" AND "Diabetes Melitus" AND "Oftalmoscopia direta" AND "Atenção Básica de Saúde”.

\section{Discussão}

No Brasil, as DCNT foram responsáveis por $56,9 \%$ de mortes no ano de $2017^{10}$, afetando principalmente a população mais desfavorecida socioeconomicamente ${ }^{10,11,14}$. No entanto, devido à grande atuação do SUS e a expansividade de atendimento da Atenção Básica, que atualmente, cobre cerca de $60 \%$ da população brasileira, houve uma redução de $20 \%$ nas taxas de óbitos pelas DCNT em território nacional. ${ }^{11}$

Devido aos processos de transição demográfica, nutricional e a epidemiológica no país, houve uma elevação dos casos e das desordens das DCNT ${ }^{1-3,10-14}$, principalmente em relação a HAS e ao DM. Atualmente esses dados são alarmantes entre as mulheres e os idosos, sendo que esse último grupo é o reflexo do envelhecimento populacional crescente, pois percebe-se um aumento da expectativa de vida da população. ${ }^{3}$ Além disso, percebe-se que os pacientes que são portadores dessas doenças sejam de forma isolada ou associadas acabam tendo como estabelecedores de risco e também de complicações, muitas delas severas, o seu estilo de vida, sendo que no país, há o destaque para o tabagismo e a inatividade física. ${ }^{11,14}$

De acordo com a Sociedade Brasileira de Endocrinologia, a prevalência de Diabetes Mellitus no país, de acordo o último censo de 2019 , é 16,8 milhões de pessoas diagnosticadas, seja pelo diabetes gestacional, pela destruição autoimune das doenças beta-pancreáticas produtoras de insulina ou pela resistência insulínica, as quais são ainda responsáveis por $30,1 \%$ de todas as mortes prematuras. ${ }^{4} \mathrm{E}$ em relação a diretriz de hipertensão, essa patologia também é predominantemente presente em território brasileiro, afetando cerca de 36 milhões de pessoas, sendo que mais de $60 \%$ são idosos. ${ }^{6}$

Nesse estudo foi possível perceber que pelo Ministério da Saúde, para controle dos danos ocasionados pelo impacto da HAS e do DM no organismo, há uma grande necessidade da mudança de hábitos de vida, principalmente em relação a alimentação não saudável, ao tabagismo, a obesidade e ao abuso do álcool, o que é importante para o processo de prevenção e terapêutica. ${ }^{5,6,13,17}$

Como se sabe, as complicações relacionadas à hipertensão e ao diabetes estão fortemente ligadas aos fatores de risco para as DCNT, determinando um grau de invalidez e redução da qualidade de vida dos pacientes mais vulneráveis, além de elevar as chances de morte prematura., ${ }^{2,3,10-16}$ Juntas elas são responsáveis por altos custos em hospitalizações e procedimento de alta complexidade devido as suas complicações, gerando saldos deficitários aos cofres públicos brasileiros . $^{1,3,14}$

A RD é um problema de saúde mundial é a principal causa de cegueira não-infecciosa ${ }^{9}$ adquirida do adulto. ${ }^{1,9,15}$ Conforme a Diretriz Brasileira de Diabetes estima-se que há 415 milhões de pacientes diabéticos no mundo, dentre eles 93 milhões desenvolveram algum grau de $\mathrm{RD}$ e $1 / 3$ deles precisam de algum tratamento. ${ }^{5}$ A glicemia de jejum, a hemoglobina glicada ( $\mathrm{HbAlc})$ e o colesterol total do soro são considerados fatores de risco independentes para o seu desenvolvimento. ${ }^{1,5,9} \mathrm{O}$ Edema Macular (EMD) e neovascularização retiniana são os principais responsáveis pela perda irreversível da acuidade visual. ${ }^{5}$

No caso do DM tipo 1 ao diagnóstico observa-se ausência de retinopatia; após 5 anos de diagnóstico têmse $25 \%$ retinopatia diabética não proliferativa, e após 20 anos de diagnóstico há $90 \%$ retinopatia diabética, $60 \%$ retinopatia diabética proliferativa, $30 \%$ de edema macular significativo, um estágio avançando para irreversibilidade. Já no DM tipo 2 ao diagnóstico há 5\% de chances de ter retinopatia diabética não proliferativa; após 5 anos de diagnóstico esse valor sobe para $30 \%$ e após 20 anos de diagnóstico, têm-se $50 \%$ de retinopatia diabética, 10\% retinopatia diabética proliferativa, 20\% edema macular clinicamente significativo. ${ }^{21}$

Já em relação a Retinopatia Hipertensiva (RH), dentro dos preceitos da Atenção Básica à saúde é considerada um achado de alto risco, pois de acordo com uma das estratificações dos pacientes hipertensos, que é baseada no Escore de Framinghan, uma boa ferramenta para avaliar os riscos da doença cardiovascular, esse paciente tem uma chance maior que $20 \%$ de apresentar um risco cardiovascular em dez anos, ou de ter lesão de órgão alvo atrelado. ${ }^{16}$

Pelo Relatório Mundial Sobre a Visão lançado em 2019 pela OMS, cerca de 2,2 bilhões de pessoas apresentam algum tipo de deficiência visual ou cegueira, e dentre elas pode-se destacar que 1 bilhão apresentam um quadro que poderia ser reversível se prevenido recocemente. ${ }^{18}$ Isso tudo estaria atrelado as desigualdades de atendimento e aos desafios enfrentados pelos sistemas de saúde no mundo inteiro., ${ }^{1,5-7,14,18}$ Portanto, a atuação do médico da Atenção Básica viabiliza uma redução desses agravos a partir da implementação em seu atendimento da oftalmoscopia direta ${ }^{1}$, sendo um exame rápido de ser feito e com bom manejo auxilia no diagnóstico das retinopatias causadas pelo diabetes e hipertensão arterial sistêmica, evitando uma possível cegueira e sua irreversibilidade. ${ }^{1,15}$

A realização da oftalmoscopia direta é um desafio para muitos profissionais médicos em todo o mundo, pois muitos acreditam que a sua realização seja uma função apenas do Oftalmologista, faltando dessa forma 
o encorajamento e incentivo para prática e investimentos em oftalmoscópios dentro da Atenção Básica. ${ }^{1,19,20}$ Sabe-se que há um grande risco de complicações pela deficiência no diagnóstico e terapêutica do DM e HAS e o elevado potencial de desenvolvimento de lesão de órgão alvo, principalmente dos vasos da retina e formação da retinopatia. Este fato requer que o Médico da atenção primária da saúde esteja preparado para aplicação de condutas precoces e exatas, como a realização da oftalmoscopia, para viabilizar a redução de danos como a cegueira e gastos públicos com procedimentos desnecessários e proporcionar mais saúde e bem-estar a população alvo.

\section{Considerações Finais}

É sabido que dentro do âmbito nacional e mundial, há uma prevalência crescente das DCNT, destacando-se a HAS e DM, as quais devido a elevação e agravamento de suas complicações, principalmente aquelas relacionadas às lesões de órgãos alvo, acabam acarretando um dispendioso processo de cuidado e atenção a longo prazo. Além do mais, tornam-se cada vez mais as principais causas de internações hospitalares, sobrecarregando a economia na implementação de propostas terapêuticas. Portanto, como visto no presente estudo, é evidente a real necessidade da implementação do aprendizado e realização do exame de fundoscopia pelos profissionais médicos da Unidade Básica de Saúde, à fim de promover um rastreio precoce dos casos de retinopatia, tanto diabética quanto hipertensiva, proporcionando uma redução no desenvolvimento de cegueira irreversível e na improdutividade dos pacientes acometidos.

Mesmo que insipiente, a inserção da oftalmoscopia direta na Atenção Básica irá viabilizar, além de um diagnóstico eficaz de uma complicação, uma inclusão ao acesso à saúde de qualidade as populações mais desprovidas e um referenciamento mais preciso ao oftalmologista.

\section{Referências}

1. Alves AP, Santos RWV, Sobrinho EFA, Rocha SPL, Loch ACN. 1. Retinopatia em pacientes hipertensos e/ou diabéticos em uma unidade de saúde da família. Rev Bras Oftalmol 2013;73(2):108-111.

2. Vigilância de Fatores de Risco e Proteção para Doenças Crônicas por Inquérito Telefônico, Compiler. VIGITEL BRASIL 2019: Vigilância de Fatores de Risco e Proteção para Doenças Crônicas por Inquérito Telefônico. Versão eletrônica. 1st ed. Brasilia- DF: Editora MS/CGDI; 2020. 139 p. 137.

3. Malta DC, Stopa SR, Szwarcwald CL, Gomes NL, Júnior JBS, Reis AAC. A vigilância e o monitoramento das principais doenças crônicas não transmissíveis no Brasil - Pesquisa Nacional de Saúde, 2013. Rev Bras Epidemiol 2015; 18:3-16.
Endocrinologia e Metabologia; 2007 Mar 26. O que é diabetes? Disponível em: https://www.endocrino.org.br/o-que-e-diabetes/.

5. Sociedade Brasileira de Diabetes, editor. Diretriz da Sociedade Brasileira de Diabetes 2019-2020 ed. Brasil: Clannad; 2019; 12-489 p. Disponível em: https://www.diabetes.org.br/profissionais/images/ DIRETRIZES-COMPLETA-2019-2020.pdf. Acessado em: 05 de junho de 2020.

6. Sociedade Brasileira de Cardiologia, editor. $7^{\text {a }}$ Diretriz Brasileira de Hipertensão Arterial. $7^{\circ}$ ed. Rio de Janeiro: SBC; 2016.

7. Retinopatia Diabética. Brasil: Academia Nacional de Medicina; 2016 Apr 19. Retinopatia Diabética; Disponível em: https://www.anm.org.br/ retinopatia-diabetica. Acessado em: 05 de junho de 2020.

8. Souza-Dias C, editor. Manual da Residência de Oftalmologia. 1st ed. São Paulo: Manole; 2018. 720 p. ISBN: 8520450849; Disponível em: ttps:// integrada.minhabiblioteca.com.br/books/9788520461402. Acessado em: 05 de junho de 2020.

9. Guedes MF, Portes AJF, Couto Júnior AS, Nunes JS, Oliveira RCC. Prevalência da retinopatia diabética em unidade do Programa de Saúde da Família. Rev Bras Oftalmol 2009;68(2):90-5

10. Secretaria em Vigilância da Saúde, editor. Panorama da vigilância de doenças crônicas não transmissíveis no Brasil, 2018: Coordenação-Geral de Vigilância de Doenças e Agravos Não Transmissíveis, Departamento de Análise em Saúde e Vigilância de Doenças Não Transmissíveis Versão 1. 40th ed. Brasilia- DF: 2019;1-15.

11. Ministério da Saúde, editor. Plano de Ações Estratégicas para o Enfrentamento das Doenças Crônicas Não Transmissíveis (DCNT) no Brasil 2011-2022. Versão eletrônica. 1st ed. Brasilia- DF: Ministério da Saúde; 2011;1-160 p. Disponível em: https://www.gov.br/saude/pt-br

12. Ministério da Saúde, editor. Política Nacional de Atenção Básica. Versão eletrônica. 1st ed. Brasilia- DF: Ministério da Saúde; 2012. 1-114 p. Disponível em: https://www.gov.br/saude/pt-br. Acessado em: 26 de junho de 2020 .

13. Carvalho Filha FSS, Nogueira LT, Medina MG. Avaliação do controle de hipertensão e diabetes na Atenção Básica: perspectiva de profissionais e usuários. Saúde Debate. 2014; 38:265-278.

14. Pereira SEA, Costa D, Penido R, Batista ANS, Calheiros A, Ferreira GV, Tavares JW, Marins RB, Messias YJ. Fatores de risco e complicações de doenças crônicas não transmissíveis. Ciência \& Saúde 2017; 10(4):213219

15. Yin, LMS; Zhang, DMD; Ren, QMS; Su, XMS; Sun, ZMD. Prevalence and risk factors of diabetic retinopathy in diabetic patients: A community based cross-sectional study. Medicine 2019; 99(9):1-6.

16. Ministério da Saúde. Cadernos de Atenção Básica: Estratégias para o cuidado da pessoa com doença crônica - Hipertensão Arterial Sistêmica. Versão eletrônica. 1st ed. Brasilia- DF: Ministério da Saúde; 2013; 1-130 p. 37. Disponível em: https://www.gov.br/saude/pt-br. Acessado em: 27 de junho de 2020 .

17. Consolim-Colombo FM, Saraiva JFK, Izar MCO, editors. Tratado de Cardiologia: SOCESP Online. 2019. $4^{\circ}$ ed. São Paulo: Manole; 1616 p. Disponível em: https://integrada.minhabiblioteca.com.br/ books/9788520457986. Acessado em: 07 de junho de 2020 .

18. World report on vision. Geneva: World Health Organization; 2019. Licence: CC BY-NC-SA 3.0 IGO.

19. Martins TGS, Costa ALFA, Martins RV, Martins EL, Alves MR, Helene O, Schor P. Modelo para o ensino da oftalmoscopia direta. Rev Bras Ens Física. 2014;36(2):1-8.

20. Yusuf I, Yang E, Knight K, Leaver L. Direct ophthalmoscopy: teaching in primary care. The Clinical Teacher 2016;(13):235-237.

21. Ávila M, Lavinsky J, Junior CAM. Retina e Vítreo: Série Oftalmologia Brasileira. 3rd ed. Rio de Janeiro: Cultura Médica: Guanabara Koogan; 2013. 